\title{
Optimizing BER in Sensor Network by Adding Relay Nodes
}

\author{
B. Brahma Reddy and K. Kishan Rao
}

\begin{abstract}
Topology control in wireless sensor networks tries to improve the performance by reducing transmission power and by confining interference, collisions and consequently retransmissions. Network performance is assessed using LQI, RSSI, BER etc. LQI a metric introduced in IEEE802.15.4 that measure the error in incoming modulation of successfully received packets [1]. In this paper, we analyze popular topology control algorithms w.r.t weak link BER and propose a novel technique to optimize BER with additional relay nodes. We introduce additional relay nodes to make the network connected instead of increasing the power. Thus it also results in saving energy. We have also evaluated the algorithm combining with LIFE, GG and RNG algorithms and demonstrated its superiority in giving high quality network.
\end{abstract}

Index Terms-Sensor networks, interference, BER, energy optimization, topology control.

\section{INTRODUCTION}

Sensor network applications became popular due to their easy and rapid deployment processes. Designing the network for better performance, leads to obtaining the data at reduced cost, timeliness and fewer errors. Present discussion is limited to stationary networks. Topology control can play major role in reducing node interference and enhancing the performance. Sensor network applications require reliable, accurate, fault-proof and possible real-time responses. Presence of large number of nodes in the network also implies the need for suitable multiple access scheme to minimize multiple user/node interference. Currently sensors are available in Bluetooth, ZigBee, and UWB following IEEE802.15 recommendations operating at 20-250 kbps data rates [2],[3]. IEEE802.15.4 uses DSSS whereas IEEE802.15.1 uses FHSS for channel access. Topology only assists in optimizing the routing tables to find optimum path between source and destination nodes. However, at underlying physical layer, RF characteristics depend on the type of the radios and transmit power used. For example, omni-directional radio keeps radiating its signal in all directions, irrespective of direction taken for topology construction. Hence, we must consider all nodes that can cause interference whether they are present in topology or not Though the sensors use time slot technique for accessing the channel, there can be multiple piconets using the same channel and in the same area resulting interference.

Manuscript received September 7, 2012; revised November 21, 2012.

B. B. Reddy is with the Vignana Bharati Institute of Technology, Aushapur, Ghatkesar, Ranga Reddy District, Hyderabad, India -501301 (e-mail: reddybb@hotmail.com).

K. K. Rao is with the Vaagdevi College of Engineering, Bollikunta, Warangal, India-506005 (e-mail: prof_kkr@rediffmail.com).
This is similar to co-channel interference in DS-CDMA network. More number of nodes attempting to use the channel leads to more noise at the intended receiving node. In case of CSMA, nodes that are beyond communication range from transmitter, but within communication range from receiver can interfere the reception at the receiver. By minimizing the number of nodes under interference distance, co-channel interference can be optimized. Network performance is always restricted by the weak link in the network. We tried to identify the weak link in terms of BER and compare in different algorithms. We found the proposed PS algorithm [4] gives better results in all sizes of the network. In order to reduce interference distance, the nodes are preferably configured at low transmit power. We made the network homogeneous by deploying relay nodes at certain places. This strategy makes the interference distance uniform to all nodes while ensuring connectivity. We may note here, currently available algorithms maintain connectivity with different transmit power levels making the network heterogeneous.

We must consider interference-connectivity while designing RF link to sustain required BER. We consider an energy and interference efficient multi-hop wireless sensor network for our discussion. If the node are located at random places and all nodes are configured uniformly with low transmit power, obviously, there is high probability of forming an unconnected network. In such case by deploying additional nodes, unconnected nodes can be converted into connected nodes. During the source destination transmission, the data stream passes through a sequence of nodes, which are classified as two different types namely; frame nodes can originate, terminate and relay the packets whereas relay nodes simply relay the received packets. The proposed scheme is executed in two stages: In first stage, we divide each unconnected link into equal segments of minimum communication range; deploy additional (relay) nodes along the Euclidean line at regular intervals of minimum communication range. In the second stage, we optimize, $N$ the number of additional relay nodes, which can lead to the minimum average BER. It is emphasized that this BER optimization can lead to larger number of nodes/edges network compared to original network. This is in contrast to general topology control algorithms which mainly focus on reducing number of edges in order optimize interference consumption. However, the resulting super-graph must preserve connectivity of original nodes.

The remainder of this paper is organized as follows: In Section II, we present related work with a focus on topology control and optimizing interference-connectivity, and BER in Wireless ad-hoc or sensor networks. In Section III, we present a scheme 
optimize interference-connectivity. The analysis and experimental results of the proposed algorithm are given in Section IV. Finally, we conclude this paper in Section V with a summary of the work done and an outlook on future work.

Definition 1: Communication range: Distance between the transmitting node and fartherest that can receive the signal directly for a given transmit power.

Definition 2: Interference-connectivity: when node C's signal can interfere with node A's signal, preventing A's signal from being received at node $B$, it's usually assumed that node $B$ must be within node C's communication range and there is communication connectivity from $C$ to $B$. We name this interference-connectivity. [5], [6]

Definition 3: LQI: Link Quality Indicator, measures the strength/quality of the received signal. This measurement may be implemented using receiver ED (Energy Detection), a signal to noise estimation or a combination of both methods.

Definition 4: BER: The bit error rate (BER), i.e., the probability that a node $B$ successfully receives an incoming bit $\tau$, denoted by $p_{\mathrm{r}}(\tau)$, is governed by the following model: $p_{\mathrm{r}}(\tau)=\operatorname{Prob}\left[\right.$ signal power of $\tau /\left(I_{\mathrm{r}}+n_{\mathrm{r}}\right)>$ $\beta_{\mathrm{r}}$ ] where $I_{\mathrm{r}}$ is the interference experienced at $B$, which is equal to the power of other nodes' transmissions and electromagnetic signals from the environment. $n_{\mathrm{r}}$ is a random variable that equals the power of ambient noise. $\beta_{\mathrm{r}}$ is a constant determined by the modulation scheme and the transceiver sensitivity.

Definition 5: Weak Link: Highest BER link in the network

Definition 6: Network BER: Average BER of all edges in the topology.

\section{RELATED WORK}

Recent research has shown that interference can make a significant impact on the performance of wireless networks, but still fewer literatures are available on interference-aware topology control. Burkhat et al [7] point out that low node degree not always implies small interference. Li et al [8] present several algorithms to construct network topologies such that the maximum and average link/nodal interference of topology is either minimized or approximately minimized. Douglas et al [9], Indranil et al [10], Ewa [11] attempted to optimize number of neighbors to the node with varying transmit power level. Wenhui Tao et al [12] using exclusive area concept adjusted transmit power to optimize the throughput. In [13] Shashi proposed hexagonal backbone for interference optimization. L.badia [14] proposed conflicting-links to indentify the interfering nodes. R.Hekmat [15] discussed interference reduction based on neighbor attachment. Maleq Khan [16] focused on stochastic failure to select transmit power levels. Several other algorithms [17]-[22], the algorithms focused on reducing number of edges to primarily optimize energy consumption and interference reduction was not the main objective.

In Chen Wei et al [23] model the assistant nodes transmissions can cause multiple packet reception at the receiving end and there by reordering requirement. In our model all the relay nodes are in-line so that relay the same packet. So packets reach the destination in the same order. Jonathan, et. al [24] focused on identifying the additional sensor placement for repairing and ensuring the fault-tolerance with $k$-connectivity. Our model is focusing more on reducing the interference and thereby improving $B E R$ while retaining connectivity. In all the above models, transmit power of the neighbors are adjusted to optimal level and preserved the connectivity. However, in our model, we keep the transmit power of all nodes at lowest level possible and add relay nodes to reduce communication range. With this we show that inspite of increased number of nodes, interference on each edge is optimized. In present analysis we restrict potential interference from co-channel only and demonstrate that topology control does reduce interference under various measurements of interference. We will address how to minimize the average link interference and also introduce two models for node interference and for each introduced model we will study how to minimize the maximum and the average interference.

The main contributions of this paper are as follows. First of all, we define various criteria to measure the interference quality of a structure. Under these interference quality criteria, we give an efficient algorithm to construct network topologies such that the maximum link (or node), or the average interference of the topology is either minimized or approximately minimized. We then further study the average performances (in terms of their interference qualities) for DSSS and FHSS MAC schemes.

Improper selection of channel in DSSS can cause co-channel interference. Similarly, the channel-hopping sequence of each piconet is derived from the master's clock. However, a node can be in more than one piconet at the same time. Though PN pattern frequency hopping, there is possibility of some overlapping and causing co-channel interference.

ZigBee has been designed as a standardized solution for sensor and control networks. ZigBee also uses a DSSS radio signal. In the 2.4-GHz ISM band sixteen channels are defined; each channel occupies $3 \mathrm{MHz}$ and channels are centered 5 $\mathrm{MHz}$ from each other, giving a 2-MHz gap between pairs of channels.

Several studies are made to analyze interference impact among Wi-Fi, Bluetooth and ZigBee while sharing the same spectrum especially $2.4 \mathrm{GHz}$. But to our knowledge, no attempt is made to study the optimization of interference within the network through topology control.

\section{NETWORK MODEL}

We consider multi-hop wireless network, and assume that each node able to gather its own location information via GPS or several localization techniques for wireless networks. We represent a network as an undirected graph $G$ $=(V, E)$ where $V=\left\{v_{1}, v_{2}, . ., v_{n}\right\}$ is a set of nodes randomly deployed in a two-dimensional plane. Each node $v \in V$ has a unique id, $\left(v_{i}\right)=i$ where $1 \leq i \leq n$ and is specified by its location. $E$ is set of edges. Let $P_{i}=\left[p_{\mathrm{i}}{ }^{1}, p_{\mathrm{i}}{ }^{2}, \ldots, p_{\mathrm{i}}{ }^{\mathrm{m}}\right]$ be a finite 
list of increasing power levels that can be assigned to node $i$ $\in V$. We denote $p_{\mathrm{i}}{ }^{1}$ the minimum power $p_{\mathrm{i}}$ such that transmission from node $i$ reach at least one node in $\left.W_{\{i}\right\}$. Further, $p_{\mathrm{i}}^{1+1}>p_{\mathrm{i}}{ }^{1}$ for any $1=1, . ., m-1$. We define $S_{\mathrm{i}}^{1}$ as the set of nodes reachable from node $i$ with the power assignment $p_{\mathrm{i}}=p_{\mathrm{i}}{ }^{1}$ for any $\mathrm{l}=1, . . m$. We remark that $\bigcup_{l=1}^{l=m} S_{i}=V \backslash\{i\}$. For ease of notation, we define $S_{0}=\phi$.

Initially all the nodes are transmitting with maximum power and are equipped with Omni directional antenna. We assume each node can control the power of transmission to save energy consumption. Let $p\left(v_{i}, v_{j}\right)$ be the power needed to support communication from node $v_{i}$ to $v_{j}$, and we call it symmetric if $p\left(v_{i}, v_{j}\right)=p\left(v_{j}, v_{i}\right)$. The power requirement is called Euclidean if it depends on the Euclidean distance $d\left(v_{i}, v_{j}\right)$. Assuming unit disk model (UDG) maximum power a node can transmit is equal to the longest Euclidean distance among all pairs of nodes. For simplicity purpose we normalize the Euclidean distance of every pair of node with longest Euclidean distance. By topology control we have sub graph $G^{\prime}=\left(V, E^{\prime}\right)$ of $G$, in $G^{\prime}$ the node has shorter and fewer numbers of edges as compare to $G$. Power consumed by $G^{\prime} \leq G$ is implied. To compute the subgraph, we start with configuring all the nodes at lowest transmit power level. With that we compute the edges that are within communication distance. In addition, we also validate the edge as per the algorithms given below. Then we verify if the subgraph is a connected network. Incase the subgraph is not connected network, we rise the transmit power of the nodes that are not connected to next level. We repeat the process till the subgraph is a connected network. Following this, we construct subgraph for popular algorithms like GG, RNG, LMST, OTC, OTTC, XTC, FLSS and LIFE. For the subgraphs produced by each algorithm, we compute BER for weak link, Network BER.

Now, we turn our attention to the PS algorithm [4]. The algorithm works in two steps:

1) Make the network connected at selected transmit power by introducing relay nodes

2) Remove the redundant relay nodes and redundant edges formed by the relay nodes if any

As an enhancement, we further optimized the graph using LIFE, GG, RNG algorithms and compared.

In this we attempt to reduce interference through reducing interference-connectivity. For this, we assume the nodes are configured initially at the lowest transmit power level possible. At this power level we identify the edges that are within communication distance. Then in order to make the network connected, we identify the unconnected edges and sort them in ascending order. We pick up each edge from sorted list and then compute number of additional nodes required to be installed between them and their locations to make the two nodes connected. Further, we also check if the subgraph produced after adding new nodes can give a connected network of original nodes. In case of not producing connected network, we go to next edge from the list and repeat the process till a connected subgraph is produced.
Now we turn our attention to identify redundant nodes among the newly added nodes and remove them. For this purpose, we follow the greedy approach wherein we select one node at a time and remove it. If the subgraph is still a connected network of original nodes, the edge is declared redundant and removed; otherwise it will be added back. We continue this for all newly added nodes and there by producing a connected subgraph with optimal number of additional nodes.

Interference for an edge is defined as $\operatorname{Cov}(e)=\mid\{w \in V \mid w$ is covered by $d(u,|u, v|)\} \bigcup\{w \in V \mid w$ is covered by $d(v,|v, u|)\} \mid$

Theorem1: Communication can be affected even one of the nodes on edge is interfered

Proof of this is given through Lemma below.

Lemma1: There node
$w \in V \mid d(u, w)>d(u, v)$ and $d(v, w) \leq d(u, v)$

Proof: Assuming omni directional radios, uniform transmit power for all radios; node $w$ is within interference distance to $v$ while it is beyond interference distance to node $u$.

Theorem2: Node can interfere when it is not part of topology.

Proof: Assume a topology graph $w \leftrightarrow u, u \leftrightarrow v, \quad w \cdots v$ and $d(w, v) \leq$ communication range Though $w$ is not connected to $v$ in the topology, signal transmitted by $w$ is with interference distance. Omni directional radio $w$ can cause interference at $v$.

\section{Simulation}

In order to demonstrate the effectiveness of our proposed algorithm, we evaluated the performance of proposed PS algorithm [4] via extensive simulations and compared with other existing algorithms. Computational experiments have been carried out on a set of moderately sized network $(20,40$, $80,100,150,200$ nodes) with symmetric links MATLAB software.

In the first experiment was done with 20 nodes distributed in $1000 \times 1000$ grid. The topologies of these nodes have been optimized using different algorithms. We evaluated the resulting topologies for free space environment as given below:

$$
\text { For DSSS } \quad P_{e}=\frac{1}{2} \operatorname{erfc}\left(\sqrt{\frac{1}{\frac{2(k-1)}{3 N}+\frac{N_{0}}{E b}}}\right)
$$

where $(k-1)$ is number of interfering nodes, $N$ is random number of chips

For FHSS

$$
P_{e}=\frac{1}{2} \exp \left(-\frac{E_{b}}{2 N_{0}}\right)\left\{1-\left(\frac{k-1}{M}\right)\right\}+\frac{1}{2}\left(\frac{k-1}{M}\right)
$$

where $(k-1)$ is number interfering nodes, $M$ is possible hoping channels

For MFSK and OFDM

$$
P_{e} \leq(M-1) \frac{1}{2} \operatorname{erfc}\left(\frac{E b \log _{2}(M)}{\sqrt{2} N_{0}}\right)
$$


where $M$ is bank of correlators / filters

The algorithms have been studied w.r.t weak link BER and network BER and results are plotte5d. 20-node connected network with normalized transmit power 0.1 is given in Fig. 1 and connected network with same transmit power but with additional nodes is given at Fig.2. Comparison of PS Algorithm with other algorithms is given in Fig.3 \& Fig.4. We have further studied by combining LIFE, GG, and RNG algorithms with PS algorithm and results are shown in Fig.5 and Fig.6. We may notice here PS and PS-RNG coinciding. We simulated with $P_{t}=0.1 \mathrm{~mW}, N F=10 \mathrm{~dB}, F M=10 \mathrm{~dB}$, $T=290 \mathrm{k}, B W=1200 \mathrm{khz}$, Data rate $=40 \mathrm{kbps}, P N$ sequence $=15$ for DSSS and Sub carriers for $F H S S=23$.

\section{CONCLSION}

Topology control, wherein nodes adjust their transmission ranges to conserve energy and reduce interference, is an important feature in wireless ad hoc networks. Contrary to most of the literature on topology control which focuses on reducing energy consumption, in this paper we tackle the topology control problem with the goal of limiting interference as much as possible, while keeping the communication graph connected. Our approach is based on the principle of reducing communication range to uniform level for all the nodes there by transmit power is uniform for all nodes. In order to make the communication range uniform, we introduce relay nodes at certain places. The proposed approach enforces symmetry on the resulting communication graph, thereby easing the operation of higher layer protocols. To evaluate the performance of our approach, we estimate the relay nodes required and their locations that guarantee connectivity of the communication graph. As shown in the previous section, the proposed PS algorithm has clearly established improvement in weak link BER and network BER terms. However, cost of the additional nodes to be traded with the saving obtained in the above specified aspects. The improvement in BER implies better BER or throughput of the network. As given in [4], the proposed algorithm also optimizes energy consumption. In the present study, we placed the additional nodes on Euclidean line to connect the unconnected nodes. However, further optimizations are also possible by position the additional nodes at optimal places.

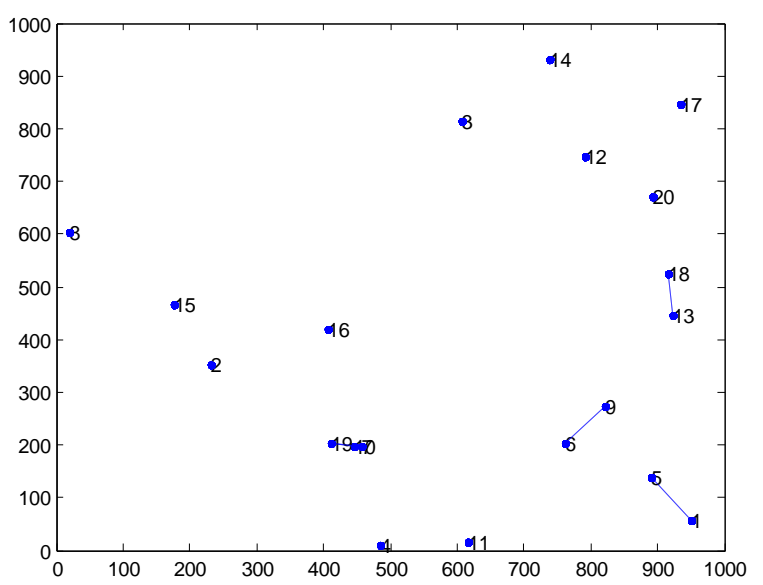

Fig. 1. 20-node unconnected network with $p=0.1$

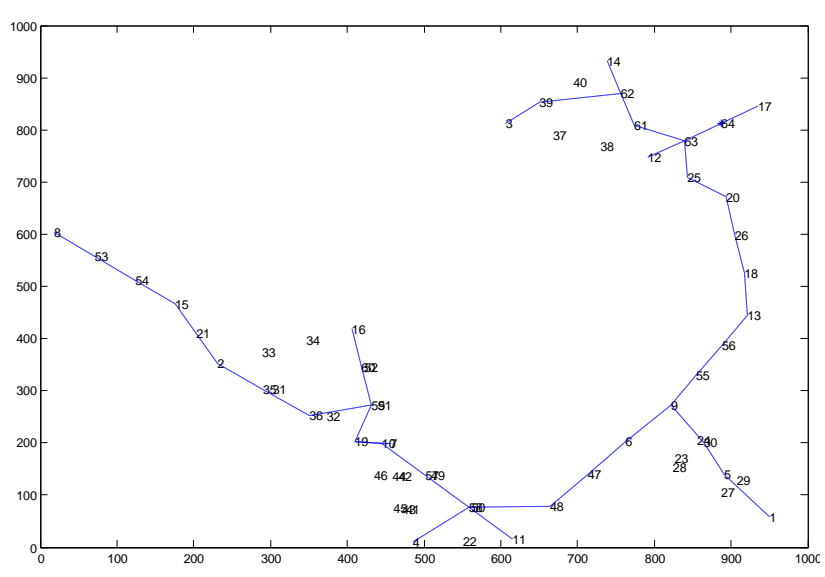

Fig. 2. 20-node connected network with $p=0.1$.

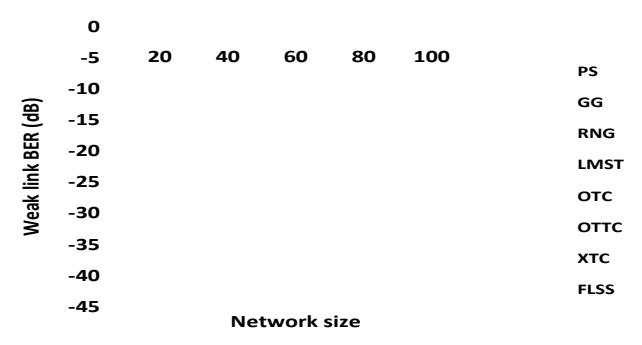

Fig. 3. Comparison of weak link BER(dB) for different network sizes.

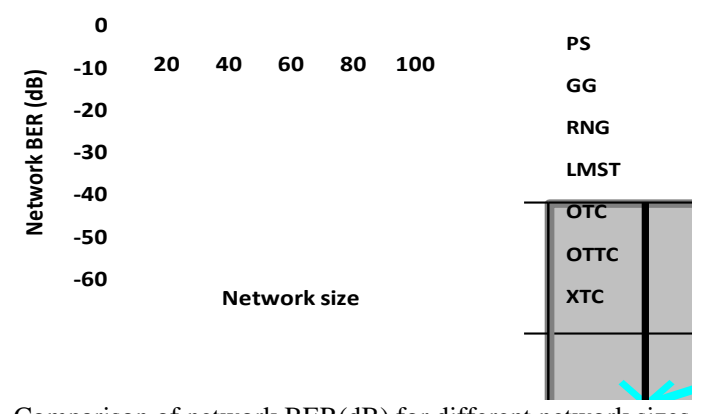

Fig. 4. Comparison of network BER(dB) for different network sizes.

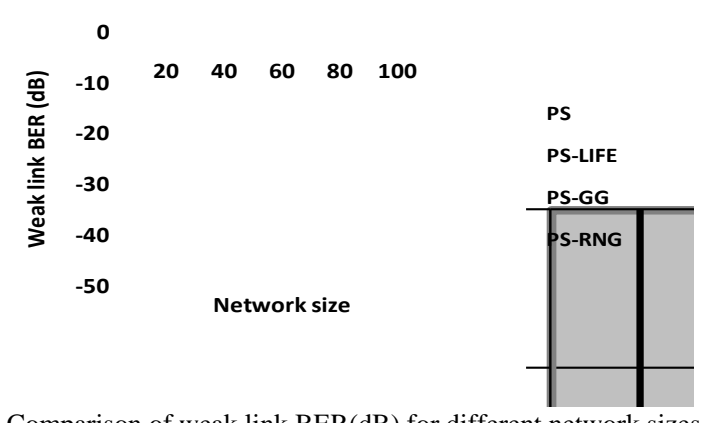

Fig. 5. Comparison of weak link BER(dB) for different network sizes.

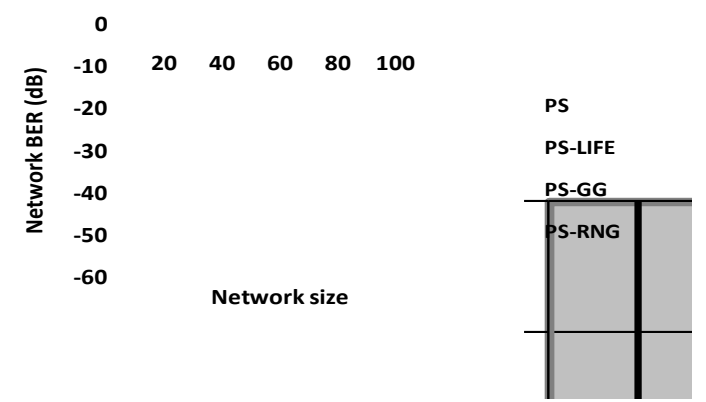

Fig. 6. Comparison of network BER(dB) for different network sizes. 


\section{REFERENCES}

[1] A. Chehri, M. P. Fortier, and H. Aniss, "RF link budget analysis at 915 $\mathrm{MHz}$ band for wireless sensor networks," World Academy of Science, Engineering and Technology, vol. 63, 2010

[2] M. U. Mahfuz and K. M. Ahmed. A review of micro-nano-scale wireless sensor networks for environmental protection: Prospects and challenges. Science and Technology of Advanced Materials 6 (2005) pp. 302-306, June 2005. [Online]. Available: http://www.elsevier.com/locate/stam

[3] J. S. Lee, Y. W. Su, and C. C. Shen, "A comparative study of wireless protocols: Bluetooth, UWB, Zig Bee, and Wi-Fi," The 33rd Annual Conference of the IEEE Industrial Electronics Society (IECON). Taipei Taiwan. Nov. 5-8, 2007,

[4] B. B. Reddy and K. K. Rao, "Deploying additional sensors with energy and interference optimization," ICWOC, Chaina, in Press, 2011.

[5] V. Rajendran, K. Obraczka, and J. J. G. L. Aceves, "Energy- efficient collision-free medium access control for wireless sensor networks," in ACM Sen Sys 2003, November 2003

[6] L. Bao and J. J. G. L. aceves, "A new approach to channel access scheduling for ad hoc networks," in ACM MobiCom 2001, pp. 210-221, July 2001.

[7] M. Bhurkhart, P. V. Rickenbanch, R. Wattenhofer, and A. Zollinger, "Does topology control reduce interference," MobiHoc'04, Roppongi, Japan. May 24-26, 2004,

[8] Li, et al., "Topology control for minimal path interference in wireless sensor networks," IEEE Symposium on Computers and Communications (ISCC 2008), 2008

[9] D. M. Blough, M. Leoncini, G. Resta, and P. Santi, “The k-neighbors approach to interference bounded and symmetric topology control in ad hoc networks," IEEE Transactions on Mobile Computing, vol. 5, no. 9, Sep. 2006

[10] I. Saha, L. K. Samasivan, S. K. Ghosh, and R. K. Patro, "Distributed fault-tolerant topology control in wireless multi-hop networks," Wireless Networks, vol. 6, no. 6, Aug. 2010

[11] N. S. Ewa, P. Kwasniewski, and I. Windyga, "Comparative study of wireless sensor networks enrgy-efficient topologies and power save protocols," Journal of Telecommunications and Information Technology, March, 2009

[12] W. H. Tao, C. Chen, B. Yang, and X. P. Guan. Adaptive Topology Control for Throughput Optimization in Wireless Sensor Networks. Technical Report. [Online]. Available: http://wicnc.sjtu.edu.cn/techreport/tao2010.pdf

[13] K. S. Prabh, C. Deshmukh, and S. Sachin, "A distributed algorithm for hexagonal topology formation in wireless sensor networks," IIT Gawahati.

[14] L. Badia, M. Mastrogiovanni, and M. Zorzi, "An Optimization framework for joint sensor deployment, Link scheduling and routing in under water sensor networks," WUW Net, Los Angeles, Sep 2006.

[15] R. Hekmat and X. An, "Relation between interference and neighbor attachment policies in ad-hoc and sensor networks," International Journal of Hybrid Information Technology, vol. 1, no. 2, April 2008.

[16] M. Khan, V. S. A. K. M. Marathe, G. Pandurangan, and S. S. Ravi, "Topology control in unreliable ad-hoc networks:Obtaining near optimal power efficiency and low interference," IEEE Journal on Select Areas in Communications, April 2009.
[17] M. Cardei, J. Wu, and S. Yang, "Topology control in ad-hoc wireless networks using cooperative communication," IEEE Transactions on Mobile Computing, vol. 5, no. 6, pp. 711-724, 2006.

[18] K. Miyao, H. Nakayama, N. Ansari, and N. Kato, "LTRT: An efficient and reliable topology control algorithm for ad-hoc networks," IEEE Transactions Onwireless Communications, vol. 8, no. 12, pp. 6050-6058, Dec. 2009.

[19] M. A. Rajan, M. G. Chandra, L. C. Reddy, and P. S. Hiremath, "Topological and energy analysis of K-connected MANETs: A semi-analytical approach," IJCSNS International Journal of Computer Science and Network Security, vol. 8, no. 2, pp. 199-206, February

[20] X. H. Liu, F. M. Li, and H. Kuang, "An optimal power-controlled topology control for wireless sensor networks," Proc. International Conference on Computer Science and Software Engineering, 2008, pp 550-554.

[21] N. K. Raya and A. K. Turuka, "Analysis of topology control alorithms in ad-hoc and sensor networks," Proc. International Conference on Challenges and Applications of Mathematics in Science and Technology (CAMIST), pp. 562-571, Jan. 2010.

[22] W. Chen, et al, "AsOR: An energy efficient multi-hop opportunistic routing protocol for wireless sensor networks over rayleigh fading channels," IEEE/ACM Transactions on Wirless Communications, vol. 8, no. 5, May 2009.

[23] G. Xing, C. Lu, Y. Zhang, Q. Huang, and R. Pless, "Minimum power configuration for wireless communication in sensor networks," ACM Transactions, Sensor Networks, vol. 3, pp. 200-233, 2007.

[24] R. Madan and S. Lall, "Distributed algorithms for maximum lifetime routing in wireless sensor networks," IEEE Transactions Wireless Communications, vol. 5, pp. 2185-2193, 2006.

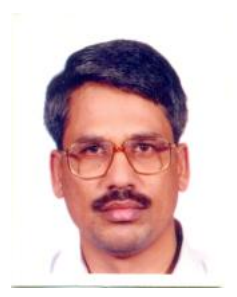

B. Brahma Reddy obtained his B. Tech from JNTU and M. Tech from IIT, Madras in 1980 \& 1982 respectively. He has worked for Indian Institute of Science, Indian Telephone Industries, National Informatics Centre, Dishnet DSL, reliance Infocomm for nearly 25 years. Past 6 years he is working as Professor in JNTU affiliated college. Currently he is perusing his Doctoral programme.

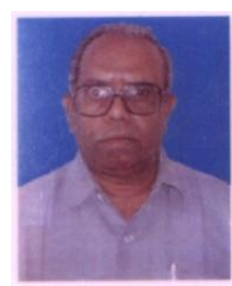

Prof. K. Kishan Rao obtained his B. E., M. E., degrees both in Distinction from O. U. College of Engineering, Osmania University in 1965 and 1967 respectively and Ph.D. from IIT Kanpur in the year 1973. Joined Regional Engineering College, Warangal in 1972 and Retired as Principal in 2002. He has Guided 3 Ph.D. Candidates, 76 M. Tech. Dissertations, 5 Research Projects and published about 68 Technical Papers in National and international Journals and Conferences. He is a Senior Member of IEEE, Life Member of IETE, Life Member of ISTE and Life Member of APSA. His Research Interest are Wireless \& Mobile Communications, Adaptive Digital Signal Processing and OFDMA Networks. Presently working as Director and Adviser to Vaagdevi Group of Technical Institutions, Warangal. 